\title{
Mesalazine for People with Diverticular Disease: A Systematic Review of Randomized Controlled Trials
}

\author{
Andrea Iannone $\mathbb{D}^{1},{ }^{1}$ Marinella Ruospo, ${ }^{2,3}$ Germaine Wong, ${ }^{4,5}$ Michele Barone $\mathbb{D}^{1},{ }^{1}$ \\ Mariabeatrice Principi $\left(\mathbb{D},{ }^{1}\right.$ Alfredo Di Leo, ${ }^{1}$ and Giovanni F. M. Strippoli ${ }^{2,4,6,7}$ \\ ${ }^{1}$ Section of Gastroenterology, Department of Emergency and Organ Transplantation, University of Bari, Italy \\ ${ }^{2}$ Diaverum Medical Scientific Office, Lund, Sweden \\ ${ }^{3}$ Department of Translational Medicine, Amedeo Avogadro University of Eastern Piedmont, Novara, Italy \\ ${ }^{4}$ Sydney School of Public Health, University of Sydney, Australia \\ ${ }^{5}$ Centre for Transplant and Renal Research, Westmead Hospital, Sydney, Australia \\ ${ }^{6}$ Diaverum Academy, Lund, Sweden \\ ${ }^{7}$ Section of Nephrology, Department of Emergency and Organ Transplantation, University of Bari, Italy
}

Correspondence should be addressed to Andrea Iannone; ianan@hotmail.it

Received 8 April 2018; Revised 12 August 2018; Accepted 30 August 2018; Published 16 September 2018

Academic Editor: Salvatore Oliva

Copyright (C) 2018 Andrea Iannone et al. This is an open access article distributed under the Creative Commons Attribution License, which permits unrestricted use, distribution, and reproduction in any medium, provided the original work is properly cited.

Background. Diverticular disease treatment is limited to fibres, antibiotics, and surgery. There is conflicting evidence on mesalazine benefits and harms. Aim. We systematically reviewed current evidence on benefits and harms of mesalazine versus all other treatments in people with diverticular disease. Methods. We searched MEDLINE, EMBASE, CENTRAL, ClinicalTrials.gov for studies published to July 2018. We estimated risk ratios (RR) for dichotomous outcomes (disease remission/recurrence, acute diverticulitis in symptomatic uncomplicated diverticular disease, need for surgery/hospitalization, all-cause/disease-related mortality, adverse events), mean differences (MD) or standardized MD (SMD) for continuous outcomes (quality of life, symptoms score, time to recurrence/remission), and their 95\% confidence intervals (CI) using random-effects models. We quantified heterogeneity by $\mathrm{Chi}^{2}$ and $\mathrm{I}^{2}$ tests. We performed subgroup analyses by disease subtype, comparator, follow-up duration, mesalazine dose, and mode of administration. Results. We identified 13 randomized trials ( $\mathrm{n}=3028$ participants). There was a higher likelihood of disease remission with mesalazine than controls in acute uncomplicated diverticulitis ( 1 trial, 81 participants, RR=2.67, $95 \% \mathrm{CI}=1.05-6.79$ ), but not in symptomatic uncomplicated diverticular disease ( 1 trial, 123 participants, $\mathrm{RR}=1.04,95 \% \mathrm{CI}=0.81$ 1.34). There was a lower likelihood of disease recurrence with mesalazine than controls in symptomatic uncomplicated diverticular disease ( 2 trials, 216 participants, $\mathrm{RR}=0.52,95 \% \mathrm{CI}=0.28-0.97)$, but not in acute uncomplicated diverticulitis ( 7 trials, 2196 participants, $\mathrm{RR}=0.90,95 \% \mathrm{CI}=0.61-1.33)$. There was no difference in the likelihood of developing acute diverticulitis in symptomatic uncomplicated diverticular disease between the two groups ( 3 trials, 484 participants, $\mathrm{RR}=0.26,95 \% \mathrm{CI}=0.06-1.20)$. There was a higher global symptoms score reduction with mesalazine than controls in symptomatic uncomplicated diverticular disease (2 trials, 326 participants, $\mathrm{SMD}=-1.01,95 \% \mathrm{CI}=-1.51,-0.52)$ and acute uncomplicated diverticulitis ( 2 trials, 153 participants, $\mathrm{SMD}=-$ $0.56,95 \% \mathrm{CI}=-0.88,-0.24)$. Conclusions. Mesalazine may reduce recurrences in symptomatic uncomplicated diverticular disease. There is uncertainty on the effect of mesalazine in achieving diverticular disease remission. Mesalazine may not prevent acute diverticulitis in symptomatic uncomplicated diverticular disease.

\section{Introduction}

The prevalence of colonic diverticulosis increases with age, ranging from approximately $30 \%$ in people aged $50-59$ years to around $70 \%$ in those older than 80 years [1]. Some $20 \%$ of people with diverticulosis experience symptoms related to diverticular mucosa inflammation, ranging from mild abdominal pain to severe complications [2]; this condition is defined diverticular disease [3]. Low dietary fibre intake, gut microbiota alteration, obesity and changes in colonic motility, and sensitivity play a role in the development of symptoms and complications in diverticular disease [4]. About $2 \%$ of 
people with diverticular disease require hospital admission, $0.5 \%$ need surgery, and $1 \%$ die during hospitalization $[5,6]$.

Current management of diverticular disease focuses on dietary and pharmacological interventions, such as rifaximin and systemic antibiotics. Some guidelines $[4,7,8]$ suggest the combination of high-fibre diet and rifaximin in symptomatic uncomplicated diverticular disease for symptoms relief and acute diverticulitis prevention. In case of acute diverticulitis, differently from early recommendations [3, 9-11], antibiotics are currently suggested only if complications arise and surgical interventions are required $[4,7,8,12-16]$. No treatment prevents diverticular disease recurrences or complications (Supplementary Table 1).

Given the existing uncertainties, mesalazine, an antiinflammatory drug, has been purported as a promising intervention in diverticular disease. Studies with different designs have investigated the use of mesalazine in diverticular disease and found contradicting results on achieving disease remission and preventing recurrences. Despite these individual conflicting findings, some clinical practice guidelines now recommend mesalazine for symptom relief in symptomatic uncomplicated diverticular disease $[7,14]$ or recurrence prevention in acute uncomplicated diverticulitis [12].

We systematically reviewed the totality of evidence on the benefits and harms of mesalazine versus all other treatments in people with diverticular disease, to inform clinical decisions.

\section{Materials and Methods}

The review was conducted according to the Preferred Reporting Items for Systematic Reviews and Meta-Analyses (PRISMA) statement [17].

2.1. Data Sources and Searches. A comprehensive literature search for randomized controlled trials of mesalazine in diverticular disease was performed in MEDLINE (1946 to July 2018), EMBASE (1996 to July 2018), the Cochrane Central Register of Controlled Trials (CENTRAL, from inception to July 2018), and ClinicalTrials.gov (searched on July 2018) using highly sensitive search strategies designed by an information specialist (RM). Searches were performed without language restriction. Relevant studies were also searched from reference lists of identified trials and guidelines. Search strategies are outlined in Supplementary Table 2.

2.2. Study Selection. Two reviewers (AI, MR) independently screened the searches by title and abstract, then the full text, to identify potentially eligible trials. We included any randomized controlled trials which compared mesalazine, at any dose, mode, and duration of administration with any intervention for the treatment of people with diverticular disease. Diverticular disease was defined as the presence of any colonic diverticula-related symptoms [3]. Considering the lack of a standardized definition of symptomatic uncomplicated diverticular disease, we considered this subtype of diverticular disease as the occurrence of nonspecific diverticular disease-related abdominal symptoms, such as lower abdominal pain and altered bowel habit, in the absence of macroscopic colonic mucosa alterations [4, 7 , 14]. Acute uncomplicated diverticulitis was defined as the presence of macroscopic diverticula inflammation which causes symptoms, such as fever, lower abdominal pain, and leukocytosis in people with diverticular disease $[4,8,14$, 15]. The development of complications, such as abscess, perforation, obstruction, or bleeding, characterized by acute complicated diverticulitis [2]. We included studies in which colonic diverticulosis diagnosis was performed by endoscopy and/or radiologic imaging.

We excluded trials not involving any arm treated with mesalazine alone or comparing this treatment only with a combination therapy including mesalazine. We also excluded trials investigating mesalazine in people with segmental colitis associated with diverticulosis, a chronic inflammatory process involving the interdiverticular mucosa, since it is considered a distinct pathologic entity $[4,18]$.

2.3. Data Extraction and Quality Assessment. Two reviewers (AI, MR) independently assessed each randomized controlled trial for eligibility and extracted data on study, population, intervention (experimental and comparator treatments), and outcomes characteristics.

2.3.1. Outcomes Measures. We evaluated the number of participants achieving diverticular disease remission, developing diverticular disease recurrence, and experiencing acute diverticulitis in symptomatic uncomplicated diverticular disease. We considered disease remission as the disappearance of all diverticula-related symptoms after the beginning of treatment with mesalazine or other interventions; relapse of diverticula-related symptoms in asymptomatic participants, after the beginning of the treatment with mesalazine or other interventions, was defined as disease recurrence.

Other outcomes were quality of life and symptoms relief assessed by any instrument reported in the trials, the number of participants requiring surgery and needing hospitalization, all-cause and diverticular disease-related mortality, any adverse events and time to diverticular disease recurrence, diverticular disease remission, and surgery and acute diverticulitis onset in symptomatic uncomplicated diverticular disease. We extracted outcome data according to the maximum period of observation in each trial.

If published outcome data were not reported or provided in sufficient detail, an author (AI) contacted trial investigators one time by electronic mail requesting any relevant additional information. When obtained, information was included in the analyses.

2.3.2. Risk of Bias Assessment. We assessed the study level risk of bias with the Cochrane risk of bias tool, including the domains of random sequence generation, allocation concealment, blinding of participants or investigators, blinding of outcome assessment, completeness of outcome data, selective reporting, and other threats to validity. 
Review authors resolved any disagreement in data extraction and quality assessment through discussion and involvement of an arbitrator (GFMS).

2.4. Data Synthesis and Analysis. We estimated risk ratios (RR) for dichotomous outcomes and mean differences (MD) for continuous outcomes, with their $95 \%$ confidence intervals (95\%CI), in individual studies. If continuous outcomes were measured using different scales across individual studies, we calculated standardized mean differences (SMD) with 95\%CI. We assessed pooled estimates using the DerSimonian and Laird random-effects model [19].

We formally estimated heterogeneity of intervention effects among studies with the $\mathrm{Chi}^{2}$ (Cochran Q) and the $\mathrm{I}^{2}$ statistics.

We performed prespecified subgroup analyses by subtype of diverticular disease (symptomatic uncomplicated diverticular disease, acute uncomplicated diverticulitis or acute complicated diverticulitis), type of comparator (probiotics, rifaximin, systemic antibiotics, placebo, or no treatment), follow-up duration (less than 3 months, 3 to 6 months, 6 to 12 months, or more than 12 months), mesalazine dose (800 to $1600 \mathrm{mg}$, more than 1600 to $2400 \mathrm{mg}$, or more than 2400 $\mathrm{mg}$ ), and mode of administration (continuous or cyclic). All subgroup analyses were stratified by subtype of diverticular disease.

To avoid double-counting of participants in trials comparing mesalazine with more than one intervention, we divided the events and total number of participants allocated to the mesalazine arm according to the number of times that data from this group was used in each analysis. We used the same method for trials including more than one mesalazine arm, with different drug doses, dividing the events and total number of patients allocated to the comparator arm according to the number of times that data from this group was used in each analysis. We estimated differences among subgroups by the Mantel-Haenszel test. We planned to explore publication bias with funnel plots where at least 10 studies were included [20].

We rated quality of evidence according to the Grades of Recommendation, Assessment, Development and Evaluation (GRADE) approach [21].

All analyses were performed using RevMan 5.3 (The Cochrane Collaboration, Copenhagen, Denmark).

\section{Results}

3.1. Search Results. Of 245 articles identified electronically, 80 were duplicates and 131 were ineligible after abstract review (Figure 1). The remaining 34 articles were retrieved and reviewed in full text form, with 13 trials (11 publications) [22-32], enrolling a total of 3028 participants with diverticular disease, included. We received additional unpublished information from the authors of 3 trials $[24,25,29]$. Two trials published in 2007 by the same group $[24,25]$ had only 8 participants included in both studies; thus they were considered as separate trials.
3.2. Characteristics of Included Studies. Table 1 shows the characteristics of populations, intervention, and comparators in the included studies. Sample size in the trials varied from 43 to 592 . Six trials (46\%) [23-27, 30] enrolled people with symptomatic uncomplicated diverticular disease and 7 (54\%) [22, 28, 29, 31, 32] enrolled people with acute uncomplicated diverticulitis. No study included patients with acute complicated diverticulitis. Mesalazine was compared with probiotics in $1(8 \%)$ [23], rifaximin in $2(15 \%)$ [24, $25]$, placebo in $8(61 \%)[26-29,31,32]$, no treatment in $1(8 \%)$ [22], and both probiotics and placebo in $1(8 \%)$ trials [30]. No study compared mesalazine with systemic antibiotics.

3.3. Quality of Studies. The risk of bias of the 13 included trials is summarized in Supplementary Figure 1.

Random sequence generation was adequate in 7 (54\%) trials and unclear in $6(46 \%)$. Allocation concealment was adequate in 2 (15\%) studies and unclear in 11 (85\%). Blinding of participants and investigators was adequate in $8(62 \%)$ trials, inadequate in $2(15 \%)$, and unclear in the remaining 3 $(23 \%)$. Seven (54\%) trials had adequate blinding of outcome assessment, while in $6(46 \%)$ this was unclear. Analysis was by intention-to-treat in $5(38 \%)$ and both intentionto-treat and per protocol in $6(46 \%)$ trials, while this was unclear in the remaining $2(16 \%)$. Withdrawal of participants from analyses was $<10 \%$ in $7(54 \%)$ trials and $>10 \%$ in 6 (46\%).

Four (31\%) out of 13 included trials were at low risk of bias for most of the quality domains [27, 30-32].

3.4. Outcomes. Table 2 summarizes key results of the comparison of mesalazine versus control interventions for diverticular disease, by disease subtype, and the grading of evidence assessment, according to the GRADE approach. Complete results of the comparison of mesalazine versus control interventions for diverticular disease are shown in Supplementary Table 3.

3.4.1. Disease Remission. There was no statistically significant difference in the likelihood of achieving diverticular disease remission between mesalazine and control interventions ( 2 trials, 204 participants, $\mathrm{RR}=1.51,95 \% \mathrm{CI}=0.57-3.98$, and $\left.\mathrm{I}^{2}=76 \%\right)$.

3.4.2. Disease Recurrence. There was no statistically significant difference in the likelihood of diverticular disease recurrence between mesalazine and control interventions ( 9 trials, 2414 participants, $\mathrm{RR}=0.83,95 \% \mathrm{CI}=0.58-1.19$, and $\mathrm{I}^{2}=73 \%$ ) (Figure 2). One trial [22] had participants treated only for 2 months and then followed up for 48 months, leading to uncertainty on the association between mesalazine and prevention of disease recurrence. Another trial [28] enrolled only people at their first diagnosis of acute uncomplicated diverticulitis, a condition with an increased response to mesalazine [2]. 


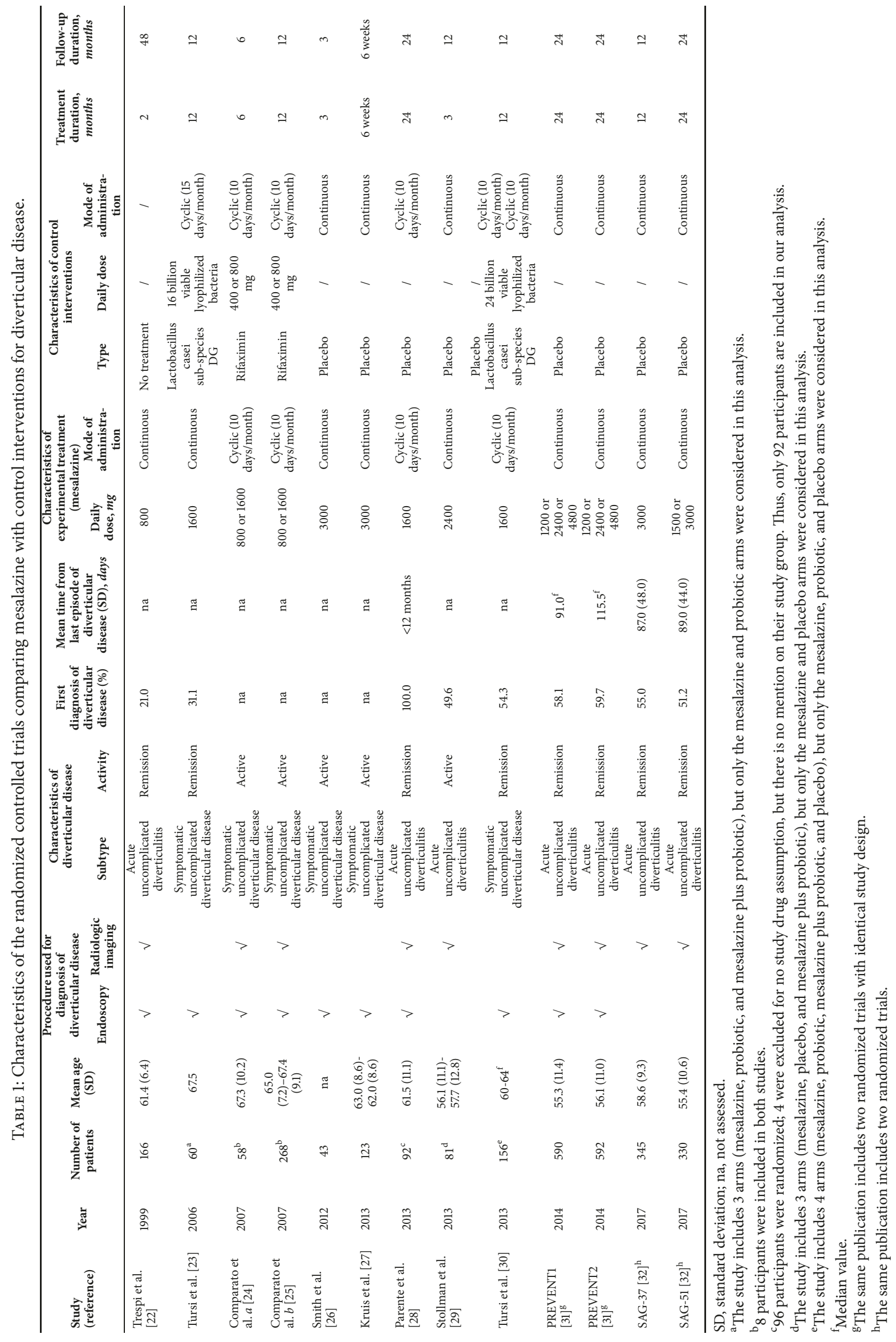




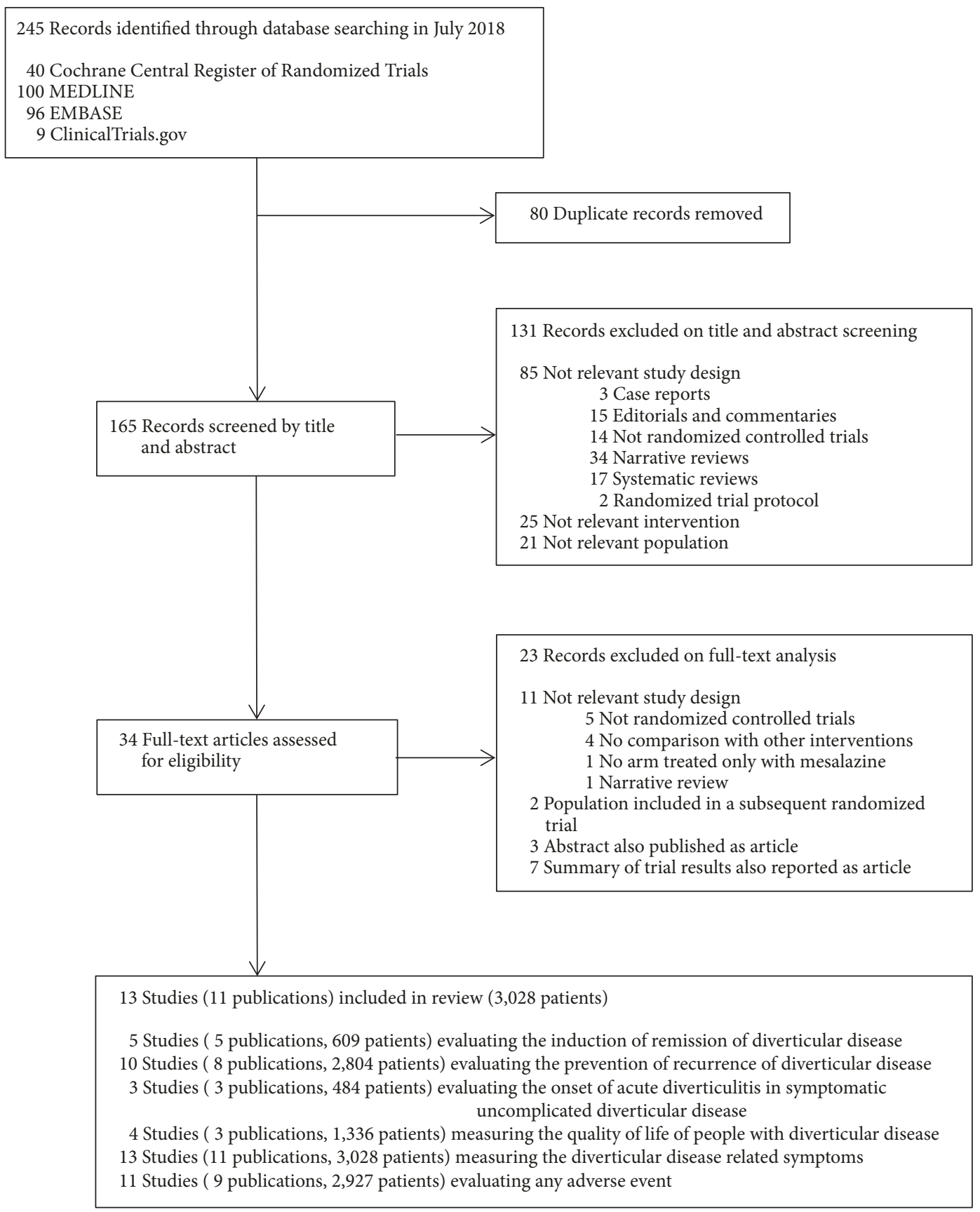

FiguRE 1: Flow diagram of search results and selection of included studies.

3.4.3. Acute Diverticulitis Onset in Symptomatic Uncomplicated Diverticular Disease. There was no statistically significant difference in the likelihood of developing acute diverticulitis in symptomatic uncomplicated diverticular disease between mesalazine and control interventions ( 3 trials, 484 participants, $\mathrm{RR}=0.26,95 \% \mathrm{CI}=0.06-1.20$, and $\mathrm{I}^{2}=0 \%$ ).

3.4.4. Quality of Life. Four trials [24, 28, 31] compared mesalazine with control interventions on quality of life (Table 3). One trial [24] included participants with symptomatic uncomplicated diverticular disease, while 3 [28,
31] included people with acute uncomplicated diverticulitis. There was a higher improvement of physical functioning $(p<0.05)$ and general health $(p=0.01)$ in the mesalazine than rifaximin group at 6 months in people with symptomatic uncomplicated diverticular disease [24]. Conversely, there was no significant difference in quality of life scores between the two groups in acute uncomplicated diverticulitis [28, 31].

3.4.5. Symptoms Relief. Thirteen trials [22-32] compared mesalazine with control interventions on symptoms relief (Supplementary Table 4). There was a statistically significant 


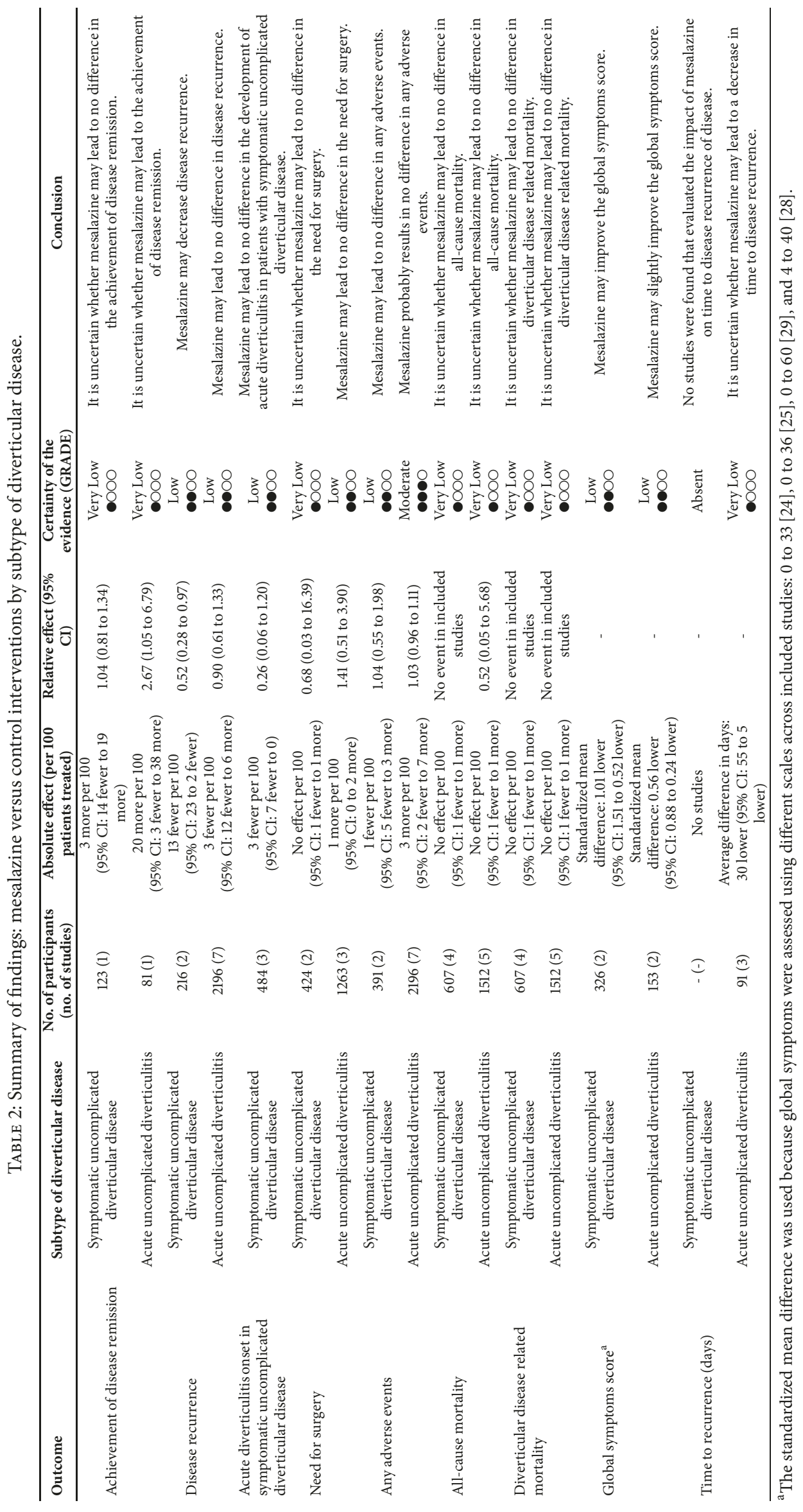




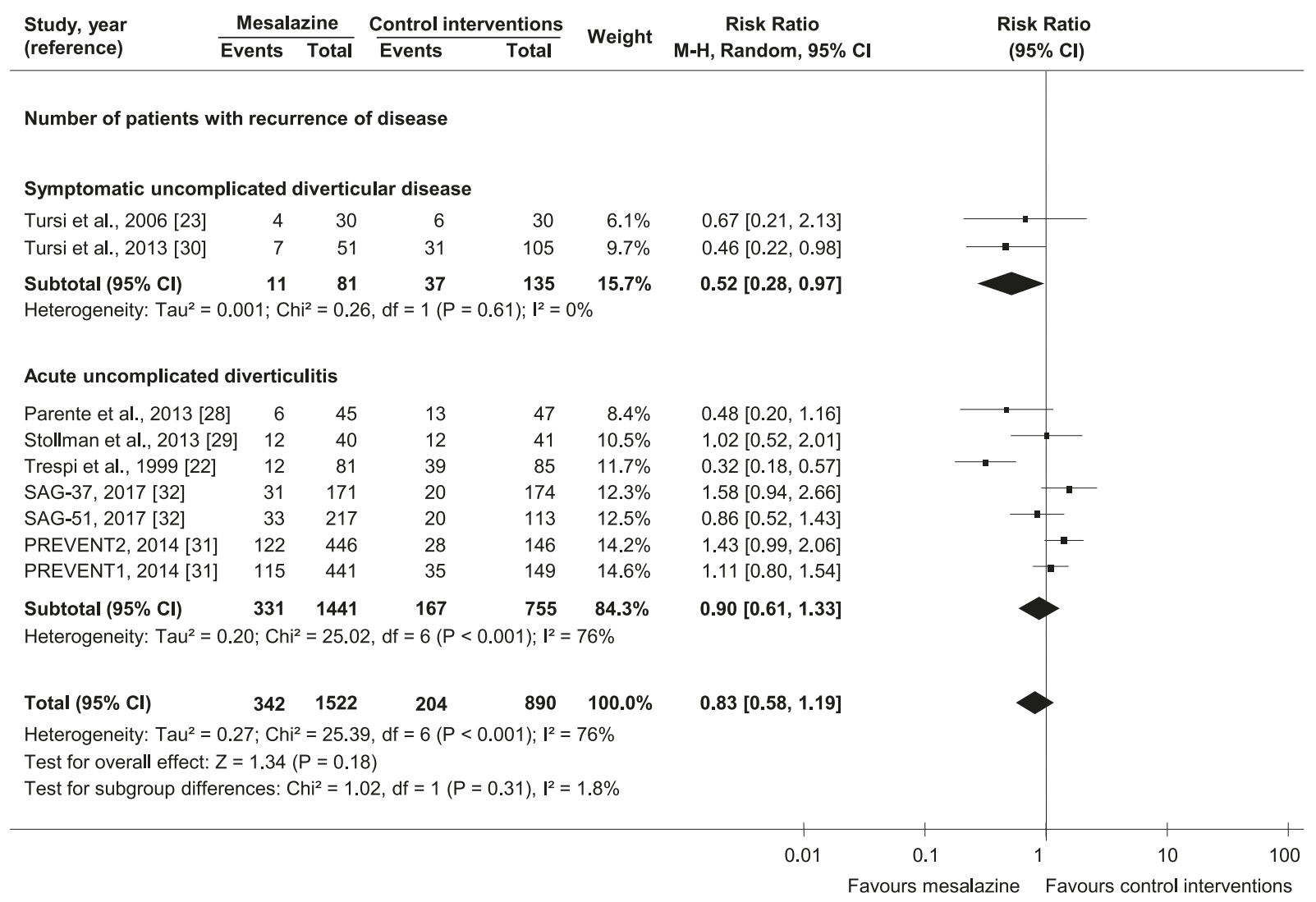

Figure 2: Comparative effectiveness of mesalazine versus control interventions by subtype of diverticular disease on the number of participants developing disease recurrence.

reduction of diverticula-related symptoms with mesalazine compared to control interventions in 4 out of 6 trials on symptomatic uncomplicated diverticular disease and in 2 out of 7 trials on acute uncomplicated diverticulitis. In the analysis of the $4[24,25,28,29]$ trials reporting a global symptoms score, there was a lower mean score at maximum follow-up with mesalazine than control interventions (4 trials, 479 participants, $\mathrm{SMD}=-0.79,95 \% \mathrm{CI}=-1.18,-0.39$, and $\mathrm{I}^{2}=72 \%$ ) (Figure 3 ). Baseline global symptoms score was not statistically different between mesalazine and control interventions arms in all included studies.

A summary of trial results for other outcomes is reported in Supplementary Table 3.

3.4.6. Subgroup Analyses. Subgroup analyses by subtype of diverticular disease, type of control intervention, follow-up duration, mesalazine dose, and mode of administration are reported in Supplementary Tables 3, 5, 6, 7, and 8, respectively.

Subtype of Diverticular Disease. There was a higher likelihood of achieving remission with mesalazine than control interventions in acute uncomplicated diverticulitis (1 trial, 81 participants, $\mathrm{RR}=2.67$, and $95 \% \mathrm{CI}=1.05-6.79$ ), but not in symptomatic uncomplicated diverticular disease (1 trial, 123 participants, $\mathrm{RR}=1.04$, and $95 \% \mathrm{CI}=0.81-1.34)$. There was no significant interaction between subgroups $(\mathrm{p}=0.06)$.
There was a lower likelihood of recurrence with mesalazine than control interventions in symptomatic uncomplicated diverticular disease ( 2 trials, 216 participants, $R R=0.52$, and $95 \% \mathrm{CI}=0.28-0.97)$, but not in acute uncomplicated diverticulitis (7 trials, 2196 participants, $\mathrm{RR}=0.90$, and 95\%CI $=0.61-$ 1.33) (Figure 2). There was no significant interaction between subgroups $(\mathrm{p}=0.14)$. There was a lower mean score at maximum follow-up with mesalazine than control interventions in both symptomatic uncomplicated diverticular disease (2 trials, 326 participants, $\mathrm{SMD}=-1.01$, and $95 \% \mathrm{CI}=-1.51,-0.52$ ) and acute uncomplicated diverticulitis (2 trials, 153 participants, $\mathrm{SMD}=-0.56$, and $95 \% \mathrm{CI}=-0.88,-0.24$ ) (Figure 3 ). There was no significant interaction between subgroups $(\mathrm{p}=0.13)$.

Type of Comparator. There was a lower likelihood of symptomatic uncomplicated diverticular disease recurrence with mesalazine only in the comparison with placebo (1 trial, 101 participants, $\mathrm{RR}=0.33$, and $95 \% \mathrm{CI}=0.13-0.86)$. There was no significant interaction among subgroups $(\mathrm{p}=0.22)$. In acute uncomplicated diverticulitis, there was a lower likelihood of recurrence with mesalazine only in the comparison with no treatment ( 1 trial, 166 participants, $R R=0.32$, and $95 \% \mathrm{CI}=0.18-0.57$ ), with a statistically significant difference among subgroups $(\mathrm{p}=0.001)$. However, in the single study [22] comparing mesalazine with no treatment participants were treated only for 2 months and then followed up for 48 


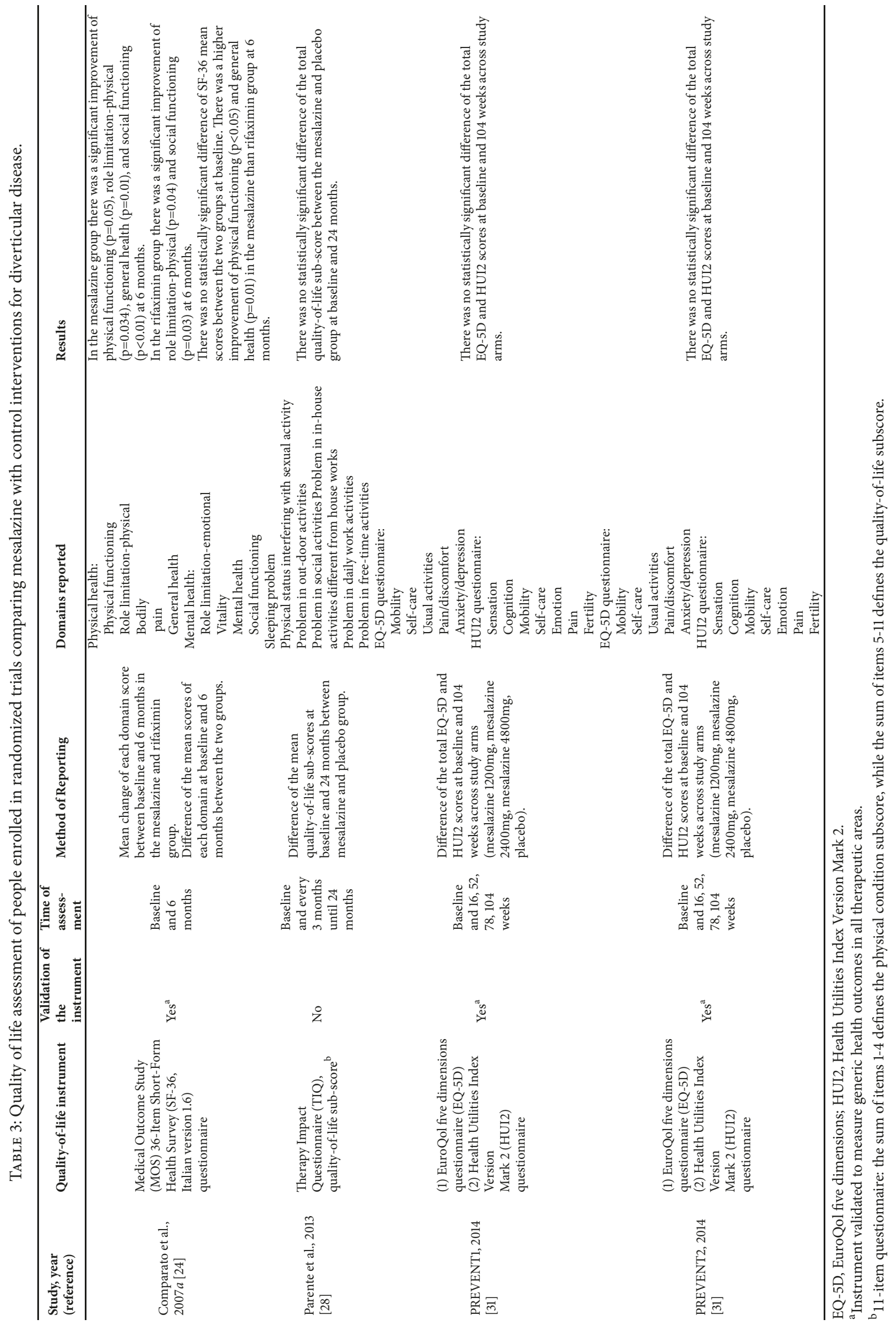




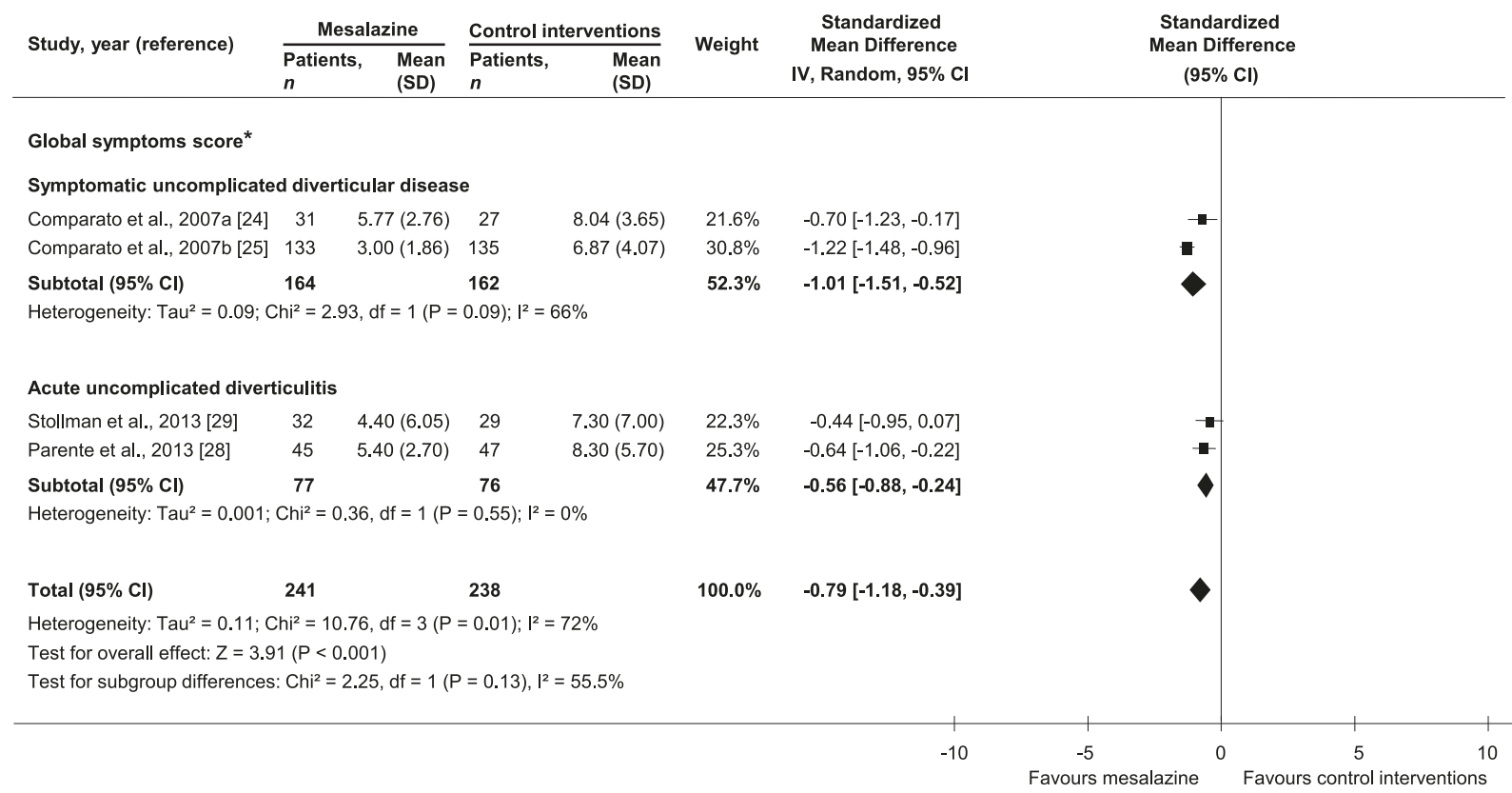

FIGURE 3: Comparative effectiveness of mesalazine versus control interventions by subtype of diverticular disease on global symptoms score. * There was not statistically significant difference in baseline global symptoms score between mesalazine and control interventions arms in all included studies.

months, leading to uncertainty on the association between mesalazine and prevention of disease recurrence.

Follow-Up Duration. In acute uncomplicated diverticulitis, there was a lower mean global symptoms score with mesalazine than control interventions only at long-term (more than 12 months) follow-up (1 trial, 92 participants, $\mathrm{SMD}=-0.64$, and $95 \% \mathrm{CI}=-1.06,-0.22)$. There was no significant interaction among subgroups $(\mathrm{p}=0.55)$.

Mode of Mesalazine Administration. There was a lower likelihood of symptomatic uncomplicated diverticular disease recurrence with mesalazine than control interventions only with cyclic administration ( 1 trial, 156 participants, $R R=0.46$, and $95 \% \mathrm{CI}=0.22-0.98)$. There was no significant interaction among subgroups $(\mathrm{p}=0.61)$. of data.

Publication bias could not be assessed due to the paucity

\section{Discussion}

We found that mesalazine may decrease recurrences in symptomatic uncomplicated diverticular disease, but not in acute uncomplicated diverticulitis. Subgroup analyses confirmed an effect in symptomatic uncomplicated diverticular disease only when mesalazine was compared with placebo or cyclically administrated. Based on current evidence from randomized trials, there is uncertainty on the effect of mesalazine in achieving remission in both symptomatic uncomplicated diverticular disease and acute uncomplicated diverticulitis. The development of acute diverticulitis in people with symptomatic uncomplicated diverticular disease may not be reduced by mesalazine. Mesalazine may improve quality of life in symptomatic uncomplicated diverticular disease and the global symptoms score in both subtypes of diverticular disease, with a larger effect in symptomatic uncomplicated diverticular disease.

In acute uncomplicated diverticulitis, existing systematic reviews either suggest a role for mesalazine in symptoms relief [33, 34] and recurrence prevention [33-36] or find no evidence supporting mesalazine use in the prevention of disease relapse [37-39]. In symptomatic uncomplicated diverticular disease, three systematic reviews found that mesalazine has a role for symptoms relief $[34,36,40]$, recurrence prevention $[34,36]$, and prevention of acute diverticulitis onset $[36,40]$.

Most of these analyses primarily described single study results without performing quantitative syntheses of data $[33-36,40]$. In two of these reviews evidence is derived from both randomized trials and observational studies [33, 36], which are an inadequate study design to address intervention questions. Existing reviews also focused on one diverticular disease subtype only [33, 35, 37-40], with methodological limitations in search strategies and study selection or in outcomes analysis, considering selected outcomes rather than the full benefits-harms trade-off for mesalazine [33-40].

We found that mesalazine may not reduce acute uncomplicated diverticulitis recurrence but may possibly lead to its remission. This last finding is based only on one trial [29] enrolling a small sample of people with 1-3 previous episodes of acute diverticulitis, a condition with a high likelihood of response to mesalazine due to the low-grade intestinal fibrosis [41]. Thus, the certainty of evidence for this outcome was graded as "very low" and we could not provide any suggestions/recommendations on the use of mesalazine in 
the acute phase of acute uncomplicated diverticulitis. Our analysis confirms the role of mesalazine in symptomatic uncomplicated diverticular disease for the prevention of recurrences, but not for acute diverticulitis onset. We also found that mesalazine may produce a higher reduction in patients' symptoms compared to control interventions in both disease subtypes, with a large difference in symptomatic uncomplicated diverticular disease and a medium difference in acute uncomplicated diverticulitis.

Benchmarked with recommendations from key international guideline agencies, our findings support the more recently guidelines which do not recommend mesalazine for the prevention of acute uncomplicated diverticulitis recurrence $[4,7,14,15]$ and acute diverticulitis onset in symptomatic uncomplicated diverticular disease [4]. Guideline recommendations on mesalazine to achieve symptomatic uncomplicated diverticular disease remission are conflicting $[4,7,14]$. We found no benefit of mesalazine in this setting, although uncertainty persists since only one trial with a shortterm follow-up assessed this outcome [27]. Finally, the Italian Group on Diverticular Diseases [4] states that there is no clear evidence on mesalazine use to achieve acute uncomplicated diverticulitis remission and prevent symptomatic uncomplicated diverticular disease recurrence. We show that mesalazine may decrease recurrences in symptomatic uncomplicated diverticular disease, while there is uncertainty on its effect in achieving acute uncomplicated diverticulitis remission.

Based on the analysis of 13 randomized trials, we can suggest the use of mesalazine for prevention of symptomatic uncomplicated diverticular disease relapse, a condition with no effective therapeutic alternatives, although results from long-term high-quality randomized trials and cost-effectiveness analyses are needed before extending the indications of this drug.

To the best of our knowledge, this is the first systematic review which compares mesalazine with other interventions for all diverticular disease subtypes, with a comprehensive evaluation of benefits and harms of this treatment and a quantitative synthesis of data. We evaluated the totality of evidence, including 13 trials versus 2-8 in existing systematic reviews [33-40], and only randomized trials compared to mixed use of both cohort and randomized designs in some previous reviews.

Our study has some limitations. Most of assessed outcomes had low/very low certainty of evidence due to the small number of included trials at low risk of bias (4 out of 13) and their heterogeneity. One trial [32] was stopped due to futility before the 24-month planned follow-up, leading to possible underestimation of events in mesalazine and control interventions arms. Moderate/high heterogeneity was found in some outcome analyses, which was mainly due to the different disease subtypes of primary studies populations. Anticipating this potential source of heterogeneity, we preplanned to stratify all outcome and subgroup analyses by diverticular disease subtype. The criteria for the diagnosis of symptomatic uncomplicated diverticular disease varied across included studies, since the definition of this condition is not yet standardized. The diagnosis of diverticular disease was not standardized across included trials (use of endoscopy, radiologic imaging, or both). We could not analyze the impact of mesalazine formulations on the considered outcomes. Most included studies used $\mathrm{pH}$-dependent preparations, with a similar drug release in the colon, while only two trials [31] used the multimatrix (MMX) mesalazine formulation. We could not evaluate the effect of mesalazine by diverticula localization. More than $90 \%$ of included patients in each trial had diverticula in the left colon and results based on disease localization were not provided. We could not evaluate some relevant outcomes (times to remission, acute diverticulitis development in symptomatic uncomplicated diverticular disease, surgery, and hospitalization), as these were not reported in included trials. Finally, we could not analyze the cost-effectiveness of the suggested therapeutic approach with mesalazine for the prevention of symptomatic uncomplicated diverticular disease recurrences because no trial provided data on costs.

There remains a need for high-quality randomized trials to further investigate the role of mesalazine in diverticular disease and, especially, its effects on achieving disease remission. These studies should also better clarify the impact of mesalazine on diverticular disease-related symptoms, quality of life, and costs in symptomatic uncomplicated diverticular disease. The role of mesalazine in acute complicated diverticulitis and the comparison between its cyclic versus continuous administration require further consideration. Finally, randomized trials including populations with right-sided disease, a condition with a described different pathogenesis from the left-sided disease, are warranted to evaluate any differences in benefits and harms of mesalazine.

\section{Conclusions}

Based on the totality of evidence, mesalazine may decrease symptomatic uncomplicated diverticular disease recurrence, whereas it may not prevent acute uncomplicated diverticulitis relapse. There is uncertainty on the effect of mesalazine in achieving remission in symptomatic uncomplicated diverticular disease and acute uncomplicated diverticulitis. The development of acute diverticulitis in people with symptomatic uncomplicated diverticular disease may not be reduced by mesalazine. Mesalazine may improve quality of life in symptomatic uncomplicated diverticular disease and patients' symptoms in both subtypes of diverticular disease, with a larger effect in symptomatic uncomplicated diverticular disease.

\section{Conflicts of Interest}

The authors declare that there are no conflicts of interest regarding the publication of this article.

\section{Acknowledgments}

The authors acknowledge Ruth Mitchell, Trial Search Coordinator, for development of search strategies; Dr. Neil Stollman and Professor Francesco Di Mario for providing additional 
information; MAPI Institute (http://www.mapigroup.com) as reference for the assessment of the validation of the symptoms and quality of life tools used in included trials.

\section{Supplementary Materials}

Supplementary Table 1: recommendations from international guideline agencies about therapeutic options in diverticular disease. Supplementary Table 2: search strategies in MEDLINE, EMBASE, CENTRAL, and ClinicalTrials.gov. Supplementary Table 3: comparative effectiveness of mesalazine versus control interventions by subtype of diverticular disease. Supplementary Table 4: symptoms assessment of participants enrolled in randomized trials comparing mesalazine with control interventions for diverticular disease. Supplementary Table 5: comparative effectiveness of mesalazine versus control interventions by type of control intervention, stratified by subtype of diverticular disease. Supplementary Table 6: comparative effectiveness of mesalazine versus control interventions by follow-up duration, stratified by subtype of diverticular disease. Supplementary Table 7: comparative effectiveness of mesalazine versus control interventions by dose of mesalazine, stratified by subtype of diverticular disease. Supplementary Table 8: comparative effectiveness of mesalazine versus control interventions by mode of administration of mesalazine, stratified by subtype of diverticular disease. Supplementary Figure 1: (a) risk of bias graph: review authors' judgement about each risk of bias item presented as percentages across all included studies; (b) risk of bias summary: review authors' judgement about each risk of bias item for each included study. (Supplementary Materials)

\section{References}

[1] J. E. Everhart and C. E. Ruhl, "Burden of Digestive Diseases in the United States Part II: Lower Gastrointestinal Diseases," Gastroenterology, vol. 136, no. 3, pp. 741-754, 2009.

[2] N. Stollman and J. B. Raskin, "Diverticular disease of the colon," The Lancet, vol. 363, no. 9409, pp. 631-639, 2004.

[3] L. Kohler, S. Sauerland, and E. Neugebauer, "Diagnosis and treatment of diverticular disease: results of a consensus development conference. The Scientific Committee of the European Association for Endoscopic Surgery," Surgical Endoscopy, vol. 13, no. 4, pp. 430-436, 1999.

[4] R. Cuomo, G. Barbara, F. Pace et al., "Italian consensus conference for colonic diverticulosis and diverticular disease," United European Gastroenterology Journal, vol. 2, no. 5, pp. 413-442, 2014.

[5] F. Sopeña and A. Lanas, "Management of colonic diverticular disease with poorly absorbed antibiotics and other therapies," Therapeutic Advances in Gastroenterology, vol. 4, no. 6, pp. 365374, 2011.

[6] D. A. Etzioni, T. M. MacK, R. W. Beart, and A. M. Kaiser, "Diverticulitis in the United States: 1998-2005: changing patterns of disease and treatment," Annals of Surgery, vol. 249, no. 2, pp. 210-217, 2009.

[7] G. A. Binda, R. Cuomo, A. Laghi et al., "Practice parameters for the treatment of colonic diverticular disease: Italian Society of Colon and Rectal Surgery (SICCR) guidelines," Techniques in Coloproctology, vol. 19, no. 10, pp. 615-626, 2015.
[8] J. C. Andersen, L. Bundgaard, H. Elbrond et al., "Danish national guidelines for treatment of diverticular disease," Danish Medical Journal, vol. 59, 2012.

[9] N. H. Stollman and J. B. Raskin, "Diagnosis and management of diverticular disease of the colon in adults. Ad Hoc Practice Parameters Committee of the American College of Gastroenterology," American Journal of Gastroenterology, vol. 94, no. 11, pp. 3110-3121, 1999.

[10] T. Murphy, R. H. Hunt, M. Fried et al., World Gastroenterology Organisation Practice Guidelines: Diverticular Disease, World Gastroenterology Organisation, 2007, http://www.worldgastroenterology.org/guidelines/global-guidelines/diverticulardisease/diverticular-disease-english.

[11] J. B. J. Fozard, N. C. Armitage, J. B. Schofield, and O. M. Jones, "ACPGBI Position Statement on Elective Resection for Diverticulitis," Colorectal Disease, vol. 13, no. 3, pp. 1-11, 2011.

[12] C. S. Andeweg, I. M. Mulder, R. J. F. Felt-Bersma et al., "Guidelines of diagnostics and treatment of acute left-sided colonic diverticulitis," Digestive Surgery, vol. 30, no. 4-6, pp. 278-292, 2014.

[13] D. Feingold, S. R. Steele, S. Lee et al., "Practice parameters for the treatment of sigmoid diverticulitis," Diseases of the Colon \& Rectum, vol. 57, no. 3, pp. 284-294, 2014.

[14] W. Kruis, C.-T. Germer, and L. Leifeld, "Diverticular disease: guidelines of the German society for gastroenterology, digestive and metabolic diseases and the German society for general and visceral surgery," Digestion, vol. 90, no. 3, pp. 190-207, 2014.

[15] N. Stollman, W. Smalley, and I. Hirano, "American Gastroenterological Association Institute Guideline on the Management of Acute Diverticulitis," Gastroenterology, vol. 149, no. 7, pp. 1944-1949, 2015.

[16] M. Sartelli, F. Catena, L. Ansaloni et al., "WSES Guidelines for the management of acute left sided colonic diverticulitis in the emergency setting," World Journal of Emergency Surgery: WJES, vol. 11, p. 37, 2016.

[17] D. Moher, A. Liberati, J. Tetzlaff, D. G. Altman, and The PRISMA Group, "Preferred reporting items for systematic reviews and meta-analyses: the PRISMA statement," Annals of Internal Medicine, vol. 151, no. 4, pp. 264-269, 2009.

[18] A. A. Sheth, W. Longo, and M. H. Floch, "Diverticular disease and diverticulitis," American Journal of Gastroenterology, vol. 103, no. 6, pp. 1550-1556, 2008.

[19] R. DerSimonian and N. Laird, "Meta-analysis in clinical trials," Controlled Clinical Trials, vol. 7, no. 3, pp. 177-188, 1986.

[20] J. A. C. Sterne, A. J. Sutton, J. P. A. Ioannidis et al., "Recommendations for examining and interpreting funnel plot asymmetry in meta-analyses of randomised controlled trials," British Medical Journal, vol. 343, no. 7818, Article ID d4002, 2011.

[21] D. Atkins, M. Eccles, S. Flottorp et al., "Systems for grading the quality of evidence and the strength of recommendations I: critical appraisal of existing approaches The GRADE Working Group," BMC Health Services Research, vol. 4, no. 38, 2004.

[22] E. Trepsi, C. Colla, P. Panizza et al., "Therapeutic and prophylactic role of mesalazine (5-ASA) in symptomatic diverticular disease of the large intestine. 4 year follow-up results," Minerva Gastroenterol Dietol, vol. 45, pp. 245-252, 1999.

[23] A. Tursi, G. Brandimarte, G. M. Giorgetti, and W. Elisei, "Mesalazine and/or Lactobacillus casei in preventing recurrence of symptomatic uncomplicated diverticular disease of the colon: A prospective, randomized, open-label study," Journal of Clinical Gastroenterology, vol. 40, no. 4, pp. 312-316, 2006. 
[24] G. Comparato, L. Fanigliulo, G. Aragona et al., "Quality of life in uncomplicated symptomatic diverticular disease: Is it another good reason for treatment?" Digestive Diseases, vol. 25, no. 3, pp. 252-259, 2007.

[25] G. Comparato, L. Fanigliulo, L. G. Cavallaro et al., "Prevention of complications and symptomatic recurrences in diverticular disease with mesalazine: A 12-month follow-up," Digestive Diseases and Sciences, vol. 52, no. 11, pp. 2934-2941, 2007.

[26] J. Smith, D. Humes, K. Garsed et al., "OC-119 Mechanistic randomised control trial of mesalazine in symptomatic diverticular disease," Gut, vol. 61, pp. A51-A52, 2012.

[27] W. Kruis, E. Meier, M. Schumacher, O. Mickisch, R. Greinwald, and R. Mueller, "Randomised clinical trial: Mesalazine (Salofalk granules) for uncomplicated diverticular disease of the colon-a placebo-controlled study," Alimentary Pharmacology \& Therapeutics, vol. 37, no. 7, pp. 680-690, 2013.

[28] F. Parente, S. Bargiggia, A. Prada et al., "Intermittent treatment with mesalazine in the prevention of diverticulitis recurrence: A randomised multicentre pilot double-blind placebo-controlled study of 24-month duration," International Journal of Colorectal Disease, vol. 28, no. 10, pp. 1423-1431, 2013.

[29] N. Stollman, S. Magowan, F. Shanahan, and E. M. M. Quigley, "A randomized controlled study of mesalamine after acute diverticulitis: Results of the DIVA trial," Journal of Clinical Gastroenterology, vol. 47, no. 7, pp. 621-629, 2013.

[30] A. Tursi, G. Brandimarte, W. Elisei et al., "Randomised clinical trial: Mesalazine and/or probiotics in maintaining remission of symptomatic uncomplicated diverticular disease - A doubleblind, randomised, placebo-controlled study," Alimentary Pharmacology \& Therapeutics, vol. 38, no. 7, pp. 741-751, 2013.

[31] J. B. Raskin, M. A. Kamm, M. M. Jamal et al., "Mesalamine did not prevent recurrent diverticulitis in phase 3 controlled trials," Gastroenterology, vol. 147, no. 4, pp. 793-802, 2014.

[32] W. Kruis, V. Kardalinos, T. Eisenbach et al., "Randomised clinical trial: mesalazine versus placebo in the prevention of diverticulitis recurrence," Alimentary Pharmacology \& Therapeutics, vol. 46, no. 3, pp. 282-291, 2017.

[33] A. M. Morris, S. E. Regenbogen, K. M. Hardiman, and S. Hendren, "Sigmoid diverticulitis: A systematic review," Journal of the American Medical Association, vol. 311, no. 3, pp. 287-297, 2014.

[34] L. Gatta, N. Vakil, D. Vaira et al., "Efficacy of 5-ASA in the treatment of colonic diverticular disease," Journal of Clinical Gastroenterology, vol. 44, no. 2, pp. 113-119, 2010.

[35] Ç. Ünlü, L. Daniels, B. C. Vrouenraets, and M. A. Boermeester, "Systematic review of medical therapy to prevent recurrent diverticulitis," International Journal of Colorectal Disease, vol. 27, no. 9, pp. 1131-1136, 2012.

[36] G. Maconi, G. Barbara, C. Bosetti, R. Cuomo, and B. Annibale, "Treatment of diverticular disease of the colon and prevention of acute diverticulitis: A systematic review," Diseases of the Colon \& Rectum, vol. 54, no. 10, pp. 1326-1338, 2011.

[37] M. A. Khan, B. Ali, W. M. Lee, and C. W. Howden, "Mesalamine does not help prevent recurrent acute colonic diverticulitis: Meta-analysis of randomized, placebo-controlled trials," American Journal of Gastroenterology, vol. 111, no. 4, pp. 579-581, 2016.

[38] S. Urushidani, A. Kuriyama, and M. Matsumura, "5aminosalicylic acid agents for prevention of recurrent diverticulitis: A systematic review and meta-analysis," Journal of Gastroenterology and Hepatology, vol. 33, no. 1, pp. 12-19, 2018.
[39] F. Carter, M. Alsayb, J. K. Marshall, and Y. Yuan, "Mesalamine (5-ASA) for the prevention of recurrent diverticulitis," Cochrane Database of Systematic Reviews, vol. 10, 2017.

[40] M. Picchio, W. Elisei, G. Brandimarte et al., "Mesalazine for the treatment of symptomatic uncomplicated diverticular disease of the colon and for primary prevention of diverticulitis: A systematic review of randomized clinical trials," Journal of Clinical Gastroenterology, vol. 50, pp. S64-S69, 2016.

[41] A. Tursi and M. Picchio, "Mesalazine in preventing acute diverticulitis occurrence: a meta-analysis of randomized, controlled trials," Journal of Gastrointestinal and Liver Diseases, vol. 25, no. 3, pp. 409-410, 2016. 


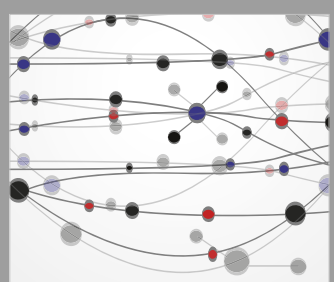

The Scientific World Journal
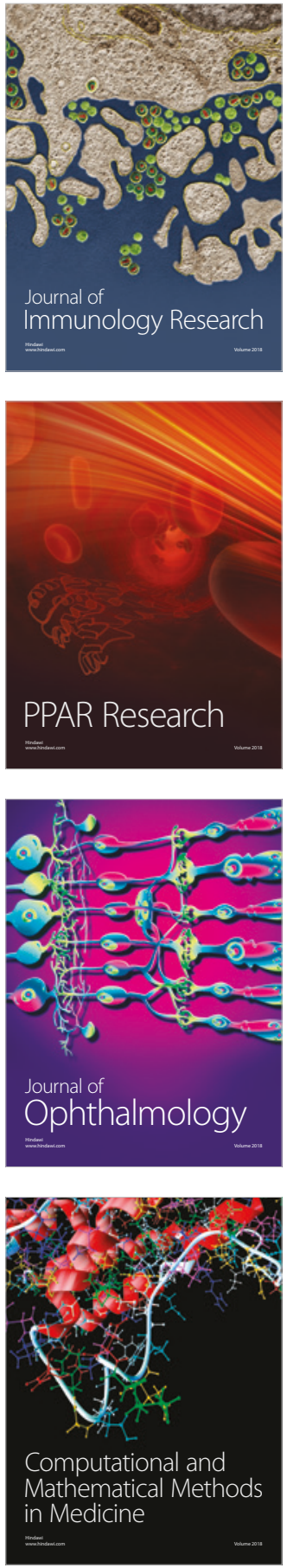

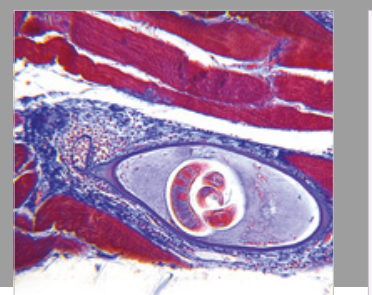

Gastroenterology Research and Practice

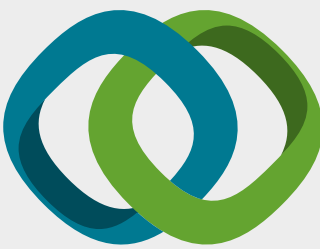

\section{Hindawi}

Submit your manuscripts at

www.hindawi.com
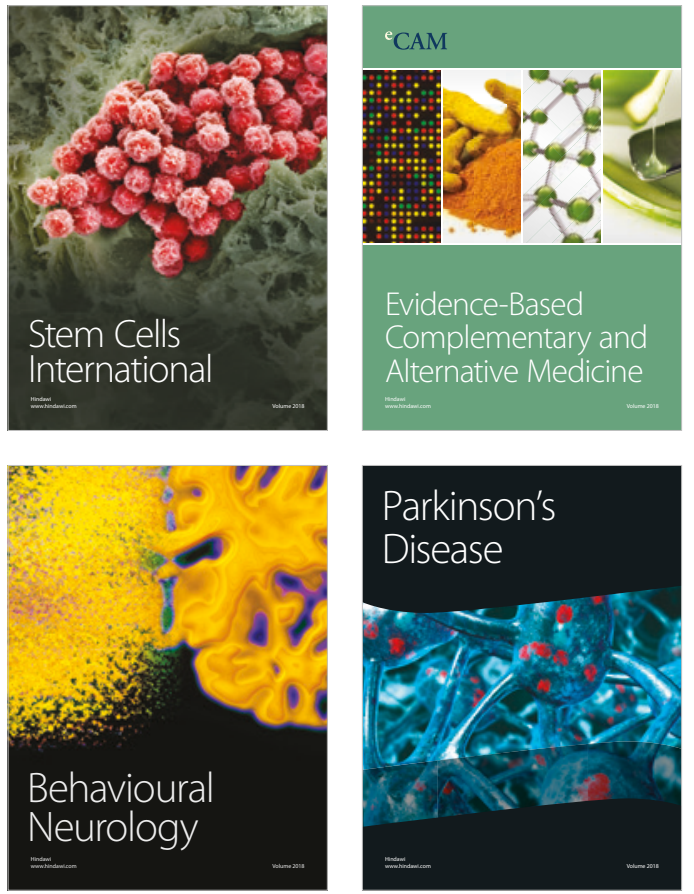

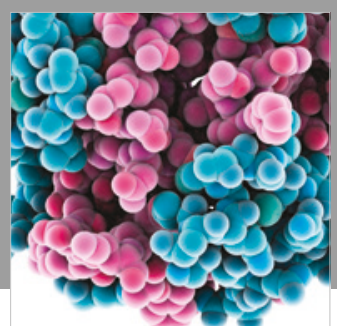

ournal of

Diabetes Research

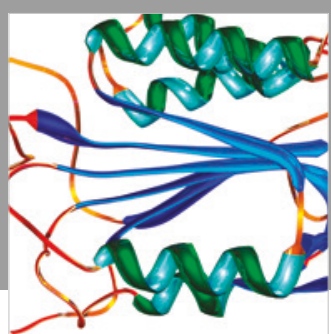

Disease Markers
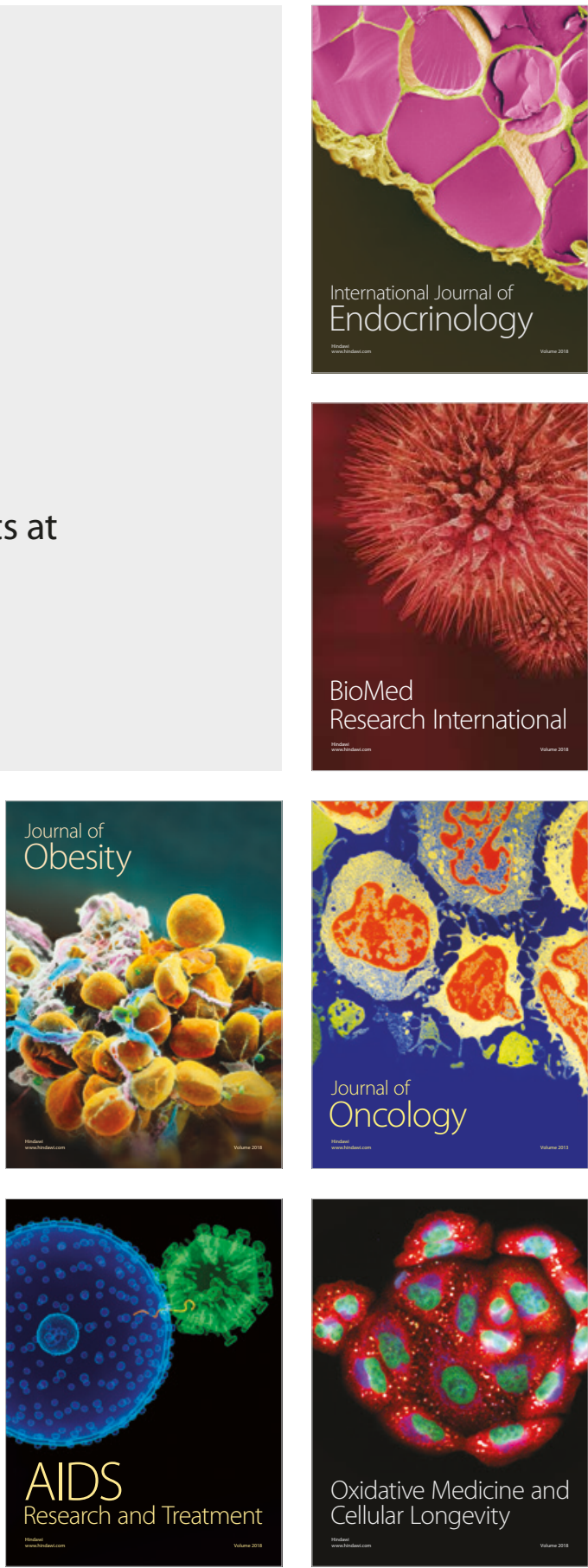